\title{
Describing, modelling and forecasting the spatial and temporal spread of COVID-19 - A short review
}

Julien Arino

\begin{abstract}
SARS-CoV-2 started propagating worldwide in January 2020 and has now reached virtually all communities on the planet. This short review provides evidence of this spread and documents modelling efforts undertaken to understand and forecast it, including a short section about the new variants that emerged in late 2020.
\end{abstract}

\section{Introduction}

On 21 February 2003, a disease now known as the severe acute respiratory syndrome (SARS) arrived in Hong Kong when a physician from Guandong Province in Mainland China bearing the SARS coronavirus (SARS-CoV), checked in at the Metropole Hotel [54]. The primary case in Hong Kong was by no means the index case: the virus had been circulating in Guandong since at least November 2002 [52]. However, that patient triggered a chain of infections that, together with earlier cases in China, led to 8,098 known cases and 774 deaths in 28 countries [42] and was declared a pandemic by the World Health Organisation (WHO).

Similarly, it is not certain at the time of writing that SARS-CoV-2 and its associated disease COVID-19 had its index case in Wuhan, Hubei Provice, China. What is certain, on the other hand, is that it is in Wuhan that COVID-19 underwent its first noticeable amplification phase, following which it spread rapidly across the world, to the point that there are now very few top level jurisdictions not having reported COVID-19 cases.

SARS-CoV-2 is the third novel Coronavirus to emerge in the 21st century (after SARS and the Middle East respiratory syndrome - MERS [28]) and the second to generate a pandemic (a third pandemic was triggered by the H1N1 influenza outbreak

Julien Arino

Department of Mathematics, University of Manitoba, Winnipeg, Manitoba, Canada, e-mail: Julien.Arino@umanitoba.ca 
in 2009). COVID-19 is also the most devastating pandemic in over a century in terms of its death toll as well as its economic and societal impact.

Here, I review some aspects of the spatio-temporal spread of COVID-19. Some caveats are in order. Firstly, while spatial epidemiology is not the most popular topic among modellers, it does remain a vast field where a myriad of approaches coexist; surveying the work done on the topic would require an entire monograph. While I have tried to be inclusive, it is certain that I have omitted some topics or techniques. I am for instance making the choice to describe mostly mechanistic models of spread, be they deterministic or stochastic, mathematical or computational. Some very good statistical work has been published on the topic of COVID-19, but I focus here to a large extent on models that can explain reality perhaps at the detriment of forecasting power. Secondly, I am aware that many modellers who have worked or are working with public health authorities may not have had the time yet to publish their work. Except for my work, I report here only on papers already published or available on recognised preprint servers. Thirdly, new variants of concern $(\mathrm{VoC})$ were detected while this paper was under review. A short section at the end of this document to describe what little is known in terms of spatio-temporal spread of these variants.

Finally, even though some work makes use of data at a very fine spatial resolution, in keeping with the philosophy of some prior work [13], I focus on models that can be used with publicly available data.

This review is organised as follows. First, in Section 2 I provide an overview of the mechanisms that lead to the spatial spread of infections and three of the major types of models that have been used to study it. In Section 3, I then describe the spread of COVID-19 from a chronological point of view. Finally, in Section 4 , I discuss modelling work specific to COVID-19.

\section{Spatialised infections - mechanisms and models}

Before considering work specific to COVID-19, let me spend some time on the spatialisation of infectious diseases in general. Indeed, while COVID-19 presents specific challenges, it is by no means the first spatial epidemic that humanity is confronted to; for instance, a simple description of the spatio-temporal trajectory of the Plague of Athens can be found in the History of the Peloponnesian War [176], which was written almost 2,500 years ago; on a more local scale, spatial epidemiology can be traced to the cholera epidemic of London in 1854 [171]. There is therefore much understanding to be gained about the current crisis by considering what was known prior to its start. 


\subsection{How does an infectious disease become spatial?}

Different conceptual models explain the mechanisms that lead to the spatialisation of an infectious disease, leading to potentially different modelling paradigms.

Working at the level of the individual, one can envision spatial spread as the repetition of inter-individual spread events. Individuals are mobile in space and it is their movement while bearing the infectious pathogen that leads to the disease becoming spatial, when they come into contact with susceptible individuals who are also mobile. This description falls mostly into what have been called Markovian contact processes [146]. When considered at the population level, this leads to models using partial differential equations and is particularly appropriate for describing the spread of a disease where the hosts can move freely, such as epizooties. Such a description can also lead to network or agent-based models.

Work with my collaborators usually instead focuses on locations and adopts a vision of spatial spread articulated in [17]: an infectious disease becomes spatialised by the repetition of processes summarised as importation, amplification, exportation and transport. Importation itself is the event when an individual infected with the disease reaches a new location. Importation is successful if the imported case leads to at least one local transmission event. This way of thinking about spread is easy to reconcile with data, since locations are jurisdictions in the context of public health. It also matches the cones of resolution that some geographers use when they think about the spatial spread of epidemics; see [11] and references therein. See also [110, 111], which consider the roles of the different levels of mobility on the spread of SARS in and to and from Beijing.

\subsection{What are the main drivers of spatial spread?}

Whatever the way one conceptualises the spatialisation process, the main driver of spatial spread is human mobility. Long range fast movements using air travel have considerably changed the way diseases spread and while amplification in a location remains driven by population effects, the initial spread is to a large extent driven by air travel. This was shown for SARS [42], the 2009 H1N1 influenza pandemic [119] and MERS [90], for instance. Long distance high speed train travel has also been associated to spread; see, e.g., [47]. It is interesting to note, though, that despite the highly heterogeneous nature of spatio-temporal spread brought on by modern travel modalities, continental-level effects can still be observed [93].

\subsection{How does one model a spatialised infectious disease?}

As mentioned in the Introduction, I focus here on mechanistic models. There are many ways to model the spatio-temporal spread of infectious diseases. Let me present 
the main contenders; see an interesting and more complete list in [161] or [147]. I do not detail reaction-diffusion equations, because, to the best of my knowledge, they have seen very little use in modelling the spatio-temporal spread of COVID-19; readers are referred to [158], for instance, for more details on deterministic aspects involving such systems. See [145] for a seminal review of the link between stochastic and deterministic spatial models, as well as interesting overviews in [61].

\subsubsection{Agent-based models}

Agent-based models (ABM) consider populations of autonomous agents that interact following some rules [76]. Agents have a set of characteristics that can be modified through their interactions with other agents. Although there are some attempts to mathematise some of the properties of such systems, they remain for the most part computational tools that need to be studied using a large number of simulations. Their strength lies in their realism: an agent can be given realistic behavioural characteristics (schedule, place of residence or of work, etc.). ABM are also easier to implement because they require very little mathematical background. Agent-based models have proved most useful when considering the effect of individual behaviours on the spread of infection in smaller populations. For instance, they have been used to study individual protective behaviour [116], the effect of presenteism while infected with a disease [126], the risk in small isolated communities [49, 129], the effect of social distancing [169] or the role of avoidance behaviour when vaccines have low effectiveness [177]. Examples of spatialised problems (all about influenza) that were studied using agent-based models include its spread in slums of Delhi [5], the use of a hybrid approach involving networks to describe the social structure and ABM to describe inter-individual spread in Forsyth Country, NC [98], the potential for social structure to generate inequalities in incidence in different areas of a county [127] or the spread within an airport terminal [165]. See also [154, 155], which use a detailed location survey to conduct a simulation of the spread of influenza in Japan. ABM are also useful as a means to model evolution; see, for instance, [96], where an ABM is used to model evolution of virulence at the front line of a spreading epidemic

The area where ABM have proved most informative is when considering spread of infections within areas where movement is constrained and generalised contact is impossible, such as buildings or cruise ships. For instance, when considering nosocomial infections, it is possible to monitor health care personnel movement and use this data to parametrise an ABM of spread within a hospital [109], or to formulate a model of spread between beds within an intensive care unit [109].

However, ABM lose in value when populations become larger, except in rare instances where unexpected emergent behaviour occurs. Where the law of large number applies, it is indeed less computationally onerous to use "classic" deterministic or stochastic models. For instance, the model in [18] reproduces almost exactly the behaviour of the agent-based model in [134], but furthermore gives access to explicit expressions of the basic reproduction number $\mathcal{R}_{0}$ and the final size of the epidemic. See also the comparisons in [7, 66]. 
Altogether, agent-based models are powerful tools of investigation at the hyperlocal scale. Considering agents consisting of groups of individuals instead of individuals also allows to operate at a higher spatial scale, although models then lose some of the interesting properties they have at the finer scale.

\subsubsection{Network models}

Network models are very similar to agent-based models, of which they were, essentially, the inspiration. The two types of models are sometimes difficult to distinguish. In epidemiology, work on networks and ABM was popularised in particular by the NIGMS Models of Infectious Disease Agent Study, which led for instance to EpiSimS [56, 77]. In network models, nodes are typically simpler than agents in agent-based models; the most straightforward example would be a network consisting of nodes (individuals) that can be in two states: susceptible to the disease or infected and infectious with it. If there is an edge in the network between two nodes, this means the two nodes came into contact; in the case of a network used to model disease spread, this indicates that a contact took place, which could lead to the transmission of the disease. One promising direction of research that has been explored using networks is that of the link between network structure and shape of the epidemic curve; see, e.g., [48, 57]. This is particularly important during the early spread of a disease and has been considered in a variety of contexts using network models.

Because they are simpler than ABM, network models are more amenable to analysis; see, e.g., [34, 38]. Originally, tools used to study the dynamics of network epidemic models originated in statistical mechanics [139, 140]. Because networks allow to incorporate a more realistic description of the contact process while maintaining some level of analytic tractability, comparing their dynamics with that of classical models is useful. In [8], this is done for instance for a two-strains influenza model with vaccination. [156] There has been a move lately towards characterising the dynamics of smaller networks using the properties of individual nodes rather than through distributions of these properties; see, e.g., [32].

While examples of use of network models in mathematical epidemiology abound, their use in situations that are specifically spatial are not as common. Instances include [79], who considered an SEIR model set in a lattice and simulated using a Monte Carlo process, to incorporate both stochasticity and space, [33], who consider the spread of dengue in city blocks or [82], who consider the spread of equine influenza. The latter paper illustrates the strength of the method, in that they have access to extensive data on horse movement between locations and are able to assess the effect of the topology of the networks, both for long range movement and shorter contact patterns with locations, on the spread of the infection.

Networks are also a natural candidate for considering the spread of infections using the air transportation network as a conduit. This was done for instance with SARS [42]. 


\subsubsection{Metapopulation models}

Also known as patch models, metapopulations couple together (typically similar) models, with each model encoding for the dynamics of the disease in a population and coupling representing the movement of individuals between the populations [16], the average time spent in remote locations [37] or the interactions between populations. Metapopulation models had been used computationally since the early 1970s, for instance to consider the spread of influenza within countries [83, 164]. They have known a resurgence since the beginning of the 21 st century, because, on the one hand, computing resources made simulating them easier and, on the other hand, papers such as [15, 19] showed that linear algebra techniques could be used to render the study of such systems very similar to that of their constituting systems. See [31] for a list of problems the authors identify as interesting challenges in the field.

Metapopulations are now quite popular and have been used in a variety of settings. A lot of work concerns investigation of properties of spatial models. For instance, with coauthors, I investigated the effect of lowering travel rates between locations [20] and of interconnection between a large urban centre and smaller satellite cities [22]. Other interesting issues studied include the effect of vaccination targeted at high risk areas [29], cooperation between governments on vaccination policy [123]. Geographically targeted vaccination has also been considered at smaller spatial scales; see, e.g., [26, 29, 101, 118, 121, 135]. Other spatial control issues have been considered in [41, 92, 95, 102, 122, 130, 136].

Papers addressing issues that are present also with COVID-19 have considered infection during transport [25], in particular in relation to entry screening [133] as well as exit and entry screening [132]. Exit and entry screening were also considered in [178]. Some work has also considered the effect of media-induced social distancing [87, 173].

Metapopulation models were used to consider specific diseases as well; the spread of SARS [162], age-structured contact patterns during the 2009 H1N1 pandemic [12], chikungunya [50], dengue [141], cholera [73] or malaria [14, 88, 89]; see also [160].

Standard metapopulation models are not well suited to consider the hyperlocal scale, because they assume homogeneity within the constituting units. In [12], an interesting approach is used that allows more heterogeneous contacts within patches. Similarly, in [148], the authors consider behaviour at the hyperlocal scale but still within a metapopulation model. In [7], the behaviour of an ABM is compared with that of a stochastic metapopulation model.

\section{Chronology and characteristics of COVID-19 spread}

To describe the spatio-temporal spread of COVID-19, I use the previously discussed framework of [17]. There is some discrepancy in reporting units, but to some extent, one can think along the lines of the ISO 3166 standard [114]. Global spread occurs 
between ISO 3166-1 codes (countries, dependent territories and special areas of geographical interest). Local spread within ISO 3166-1 codes occurs between ISO 3166-2 codes (provinces in Australia and Canada, départements in France, states in Brazil and the USA, etc.). Many countries also report at a finer geographical scale, which I still call local: counties, regional health authorities or cities. Anything below the city level is hyperlocal; typically, this corresponds to commuting to work, school or shopping, but can even be mobility within a building. In keeping with my avowed preference for publicly available data, my description involves higher level jurisdictions rather than spread at the hyperlocal scale, which is typically associated to confidential data.

Throughout the description that follows, one should bear in mind that data used to describe the spread is very likely wrong in some instances, or rather, some jurisdictions might be reporting with a delay because of a lower capacity to detect cases. See for instance [100], in which two health security indices, the Global Health Security Index and the Joint External Evaluation, are used to assess the likelihood that countries detected COVID-19 early. (The work also shows that countries with higher values of these indices also saw reduced mortality from the disease to 1 July 2020 , although this is likely not true anymore.)

\subsection{Chronology and characteristics of global spread}

There is evidence that COVID-19 could have started its global spread in December 2019, with reports of a case in France [68] as well as suspicious cases [39] and positive wastewater samples [128] in Italy. However, these retrospective analyses have yet to be confirmed, so at the time of writing, the first ten locations to have confirmed importations are those listed in Table 1. The remainder of January saw cases being confirmed in several other countries. Of note is that China imposed a cordon sanitaire in Wuhan on 23 January 2020 and that the first successful importation (in the terminology of [17], i.e., a local transmission event) was reported by Vietnam on 24 January 2020 [182].

Starting in February 2020 and with more and more of the locations having reported importations earlier seeing local transmission chains, global spread accelerated. Figure 1 shows the number of ISO 3166-1-alpha3 codes (top level jurisdictions) reporting their first confirmed case as a function of the time since the first confirmed international exportation event.

While I do not detail them in the modelling section because of my focus there on models able to provide explanations of the phenomena, it is worth noting that interesting time series analyses were performed during the early stages of spread. For instance, [58] used ARIMA analysis of travel data together with disease propagation data to forecast future destinations. The authors find that uncertainty as to the percentage of asymptomatic cases makes previsions complicated; this conclusion is in line with personal work [23]. Likewise, [99, 105] considered spatial autoregressive models. Also, although not global, continental-level spread as documented for Africa 


\begin{tabular}{|c|c|c|c|}
\hline Date & Location & Note & Source \\
\hline 13 Jan. & Thailand & Arrived 8 Jan. & {$[181$} \\
\hline 16 Jan. & Japan & Arrived 6 Jan. & [168, 179 \\
\hline 20 Jan. & Republic of Korea & Airport detected on 19 Jan. & {$[180$} \\
\hline 20 Jan. & USA & Arriv & 107 \\
\hline 23 Jan. & Nepal & Arrive & [35] \\
\hline 23 Jan. & Singapore & Arrived 20 Jan. & $|2|$ \\
\hline 24 Jan. & France & Arrived 22 Jan. & {$[1]$} \\
\hline 24 Jan. & Vietnam & Arrived 13 Jan. & [60, 152] \\
\hline 25 Jan. & Australia & Arrived 19 Jan. & 27 \\
\hline 25 Jan. & Malaysia & Arrived 24 Jan. & [151, 74 \\
\hline
\end{tabular}

Table 1 First ten international locations having reported imported COVID-19. Date refers to the date the case was reported. All dates are in 2020. All cases in this table were imported from China, except for Vietnam, which concerned both an imported case and a local contact.

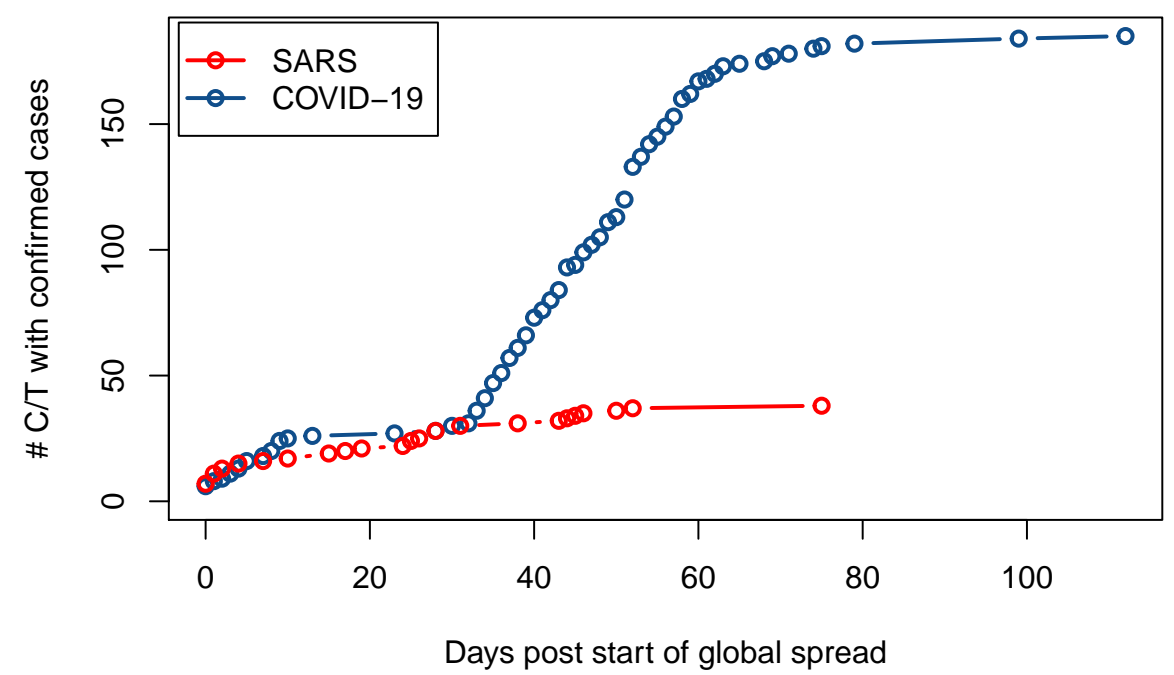

Fig. 1 Number of countries and territories (ISO-3166-1-alpha3 codes) having reported confirmed cases of SARS-CoV (red) and SARS-CoV-2 (blue) as a function of the number of days since importation in HKG (SARS-CoV) and importation in THL (SARS-CoV-2).

in [91] is included here because the focus is not on the transition between the global level to the continental level but on spread within the continent. Finally, [138] use self-organising maps to look for similarities in epidemic curves to identify countries seeing propagation of the same type.

\subsection{Attempts to slow down the global spread}

Using the terminology of the conceptual model of spatialisation, when COVID-19 started its international spread, there were very few jurisdictions that were exporters 
of COVID-19 and an immense majority of potential importers. Public health authorities in those jurisdictions that did not have cases at that point therefore took measures to try to stop or at least delay importations. To this end, they used three main types of measures: restriction or suspension of travel, entry screening and post-arrival self-isolation measures.

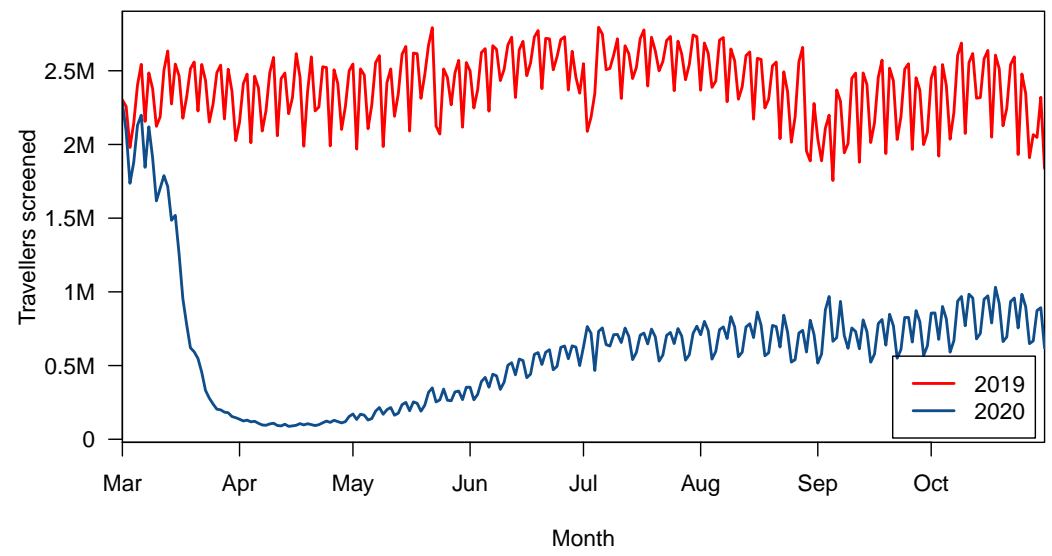

Fig. 2 Daily number of passengers processed by the United States Transport Security Agency (TSA) in 2019 and 2020; data from https://www.tsa.gov/coronavirus/passenger-throughput

Starting early on and ongoing at the time of writing, various jurisdictions took measures to curtail or even interrupt travel. Passengers themselves also abstained from travelling. The result of this was a precipitous drop in travel volumes. The intensity of this effect can be seen in Figure 2, which shows the daily number of passengers processed by the United States Transport Security Agency, i.e., the number of individuals undertaking a trip originating in the USA, in 2019 (red) and 2020 (blue). The data shown for 2019 is for a year earlier, but shifted so it corresponds to the same day in the week. At the lowest point, on Thursday 16 April 2020, TSA screened 3.63\% of the number of travellers they had screened on Thursday 18 April 2019. The same trend can be observed for instance in tourism, with the United Nations World Tourism Organisation Tourism Dashboard (https:// WwW.unwto.org/international-tourism-and-covid-19) reporting that the number of international tourists arrivals in April and May 2020 was 97\% less than the same months in 2019.

In [6], an analysis of the global air transportation network is undertaken using the network distance defined in [44], attempting to tease out the effect of travel interruptions on the spread. While both of these studies provide very interesting insights into the issue, a precise quantification of the effect of such fundamental changes to travel is hard.

Entry screening is typically implemented at ports of entry (ports, airports, border crossings) and seeks to identify individuals who are bearing the disease of concern, in order to isolate them and thereby avoid potential transmission of the disease in 
the local population. There is some debate about the usefulness of entry screening, especially during the early stages of a global spread event. See [149] for an extensive review. The sensitivity and specificity of the thermal detection equipment used is questionable [40]. In the case of COVID-19, it has also been argued that this low sensitivity would combine with the fact that fever detection would often fail because of the frequency of asymptomatic cases [45]. Entry screening at the beginning of a health crisis also means looking for a needle in a haystack, since the volume of incoming passengers from all locations vastly dominates the volume of passengers coming from the location of interest [120]. Since prevalence is low at the beginning of the event, this further compounds the lack of efficacy and results in poor characteristics for the method [67]. Also, screening protocols themselves vary widely from location to location [86], rendering a general evaluation of the value of a protocol difficult. Despite these reservations, in the case of COVID-19, some of the evidence of early international spread comes through entry screening, so there seems to have been some limited benefit to thermal imaging entry screening.

After the initial few days during which testing was thermal imaging-based, screening switched to using much more reliable PCR tests. This became possible because sequencing of the virus genome was performed remarkably quickly. As a consequence, currently, there are four main attitudes towards screening: no screening at all; "soft" screening, i.e., verbal or written questionnaires; testing on entry; testing prior to entry. Some countries use a combination of approaches, for instance requiring testing only for individuals arriving from regions considered particularly at risk.

A jurisdiction still has one option to combat the risk that successful importations take place: it can recommend or impose that individuals arriving from another jurisdiction spend some time in quarantine. Canada, for instance, has insisted on a two-weeks quarantine period for all incoming travellers since the beginning of the crisis, with exemptions.

\subsection{From global to local spread}

The first step in switching from a purely global vision of spread to a local one is to consider when COVID-19 could arrive in "one's backyard". In the early stages of the pandemic, before most top-level jurisdictions reported reported human-tohuman transmission chains, it was of interest to those jurisdictions having no or few local cases to understand the risks that their connectedness to other jurisdictions carried. Because of evidence gathered during past pandemics and other notable public health events (see Section 2.2. , this evaluation was mostly carried out by investigating a given jurisdiction's connection to the rest of the world by means of the air transportation network. This was the method used for instance in Mexico [64], India [97] or Europe [157]. Much practical work on this aspect has come to rely on global airline transportation data such as that provided by the International Air Transport Association (IATA). However, it should be noted that this dataset 
quickly became unreliable because of the dramatic fall in travel volumes discussed in Section 3.2.

\subsection{Chronology and characteristics of local spread}

Most of the very early work on local spread concerned China, since it was the first country to experience this. The same type of method was used in [78] as is detailed at the start of this section, but with past data on mobility during the Spring Festival of millions of migrant workers residing in Wuhan. The aim was to assess the risk to locations visited by the migrant workers. Since Chinese New Year was on 25 January 2020, while the cordon sanitaire was imposed in Wuhan on 23 January, a lot of individuals did make the trip. This allowed the authors to venture which places were probably under-reporting cases. See also [184], which uses GIS techniques to study the spread within China and the factors contributing to this spread. As do the authors of the previous paper, they find that connection to Wuhan, both in terms of population flow and economically, was the main driver of the initial spread.

Tracing transmission chains originating from importations allowed to better understand the consequences of importations. See, for instance, [36], which breaks down such a transmission chain that started on 27 January 2020 in Bavaria (Germany). In [46], the early spread in Brazil is documented, from importation from Europe (as evidenced by genome typing of the strains) to local spread within states, finally followed by exportation from urban centres. This is confirmed by [84], who consider spread among 604 cities in São Paulo State, Brazil. They show that in the heterogeneous setting they consider, there are two patterns of spread: one spatial, where the disease spreads to the nearest spatial component; the other hierarchical, where within one unit, spread starts with the top level urban centre then makes its way to smaller cities. In [80], the authors use genomic and transportation data to consider the spread within the USA and conclude that quite early on in the spread, importations into uninfected locations in the country were much more likely to originate elsewhere in the country than abroad. Propagation within the USA was also studied by [106], in which the occurrence of space-time clusters is studied. This interestingly shows that as the epidemic took hold, there occurred more and more smaller clusters, confirming in some sense the similar observations in Brazil. Another investigation of continental spread in the USA is carried out in [144] using a multilayer perceptron neural network. The authors use the Moran index computed on the incidence rates and a large number (57) of explanatory variables: socioeconomic, behavioural, environmental, topographic, demographic, age-adjusted mortality rates from several diseases, both infectious and chronic. They find that some of the most important factors predicting COVID-19 incidence rates are the age-adjusted mortality rates of ischemic heart disease, pancreatic cancer and leukemia, median household income and total precipitation. In [3], the time evolution in several countries and the time evolution within France are investigated using time series methods incorporating 
spatial components. While mostly methodological, this provides interesting tools to consider the spatio-temporal evolution of the disease across multiple jurisdictions.

Other authors considered mechanisms for slowing down the spatial spread within a country. The authors of [142] advocate for a disconnection between locked-down urban centres and rural areas in India as a means to avoid complete country-wide lockdown. This position is justified; indeed, authors in [63], for instance, found strong correlation between population density and the spread of SARS-CoV-2 in China, so it could be that forbidding movement between locations at high risk (the cities) and those at lower risk is a valid approach. However, to the best of our knowledge, no nation implemented such a system; indeed, during the initial wave, most countries implemented country-level lockdowns that also relied on severely limiting or completely interrupting mobility within their territory. In [70], the authors consider the effect of containment measures on the spread of COVID-19 between provinces in Italy. In [124], the authors used human mobility data in China to consider the spread of COVID-19 within China, in particular in relation to the impact of control measures.

\subsection{Chronology and characteristics of hyperlocal spread}

Hyperlocal spread was documented early on during the course of the pandemic because of cases that happened onboard cruise ships that were under quarantine. These events, while unfortunate for those involved, have provided a wealth of data. In particular, they were extremely helpful in finding out key epidemiologic parameters such as reproduction number [185], prevalence of asymptomatic infections [75], incidence [153], transmissibility of the disease [143] or case fatality ratio [163]. Because cruise ships have records of who was infected together with the room they were in, it should become possible to build a good understanding of spatial aspects, although to the best of our knowledge, this data has not yet been released.

Many countries faced and are facing outbreaks in long-term care facilities (LTC). There are a variety of reasons for this elevated risk; see, e.g., [172, 175]. This led to trememdous effort to control such outbreaks [175]. Movement within LTC can be documented (and modelled) accurately; see, e.g., [51, 150]. The health of residents is also monitored (usually) well. As a consequence, nosocomial COVID-19 outbreaks also provide valuable data at the hyperlocal level. See documented outbreaks in [113, 125, 137, 166, 167].

Note an interesting "twist" on hyperlocal spread: in [108], the authors conduct a wide-ranging analysis at the hyperlocal scale, in the sense that the consider the movements of individuals at the local scale but over the entire territory of the United States of America. This allows them to consider the effect of spatial heterogeneity of public health orders. 


\section{COVID-19-specific models}

I replicate here the hierarchical spatial structure in Section 3 rather than the methodological one in Section 2.3. Indeed, while most work detailed here falls within one of the three classes of methods in Section 2.3. I also report on other methods that gave interesting results.

\subsection{Models of global spread}

To the best of my knowledge, most models for the global spread had in their objectives to study how to slow down the global spread of the infection or considered spread within specific countries or groups of countries; these are discussed in the relevant later sections.

In [21, 24], we set the $S L_{1} L_{2} I_{1} I_{2} A_{1} A_{2} R$ model of [23] in a metapopulation context and focused on the risk of importation in different countries. The model was run daily to provide the Public Health Agency of Canada with an assessment of the most likely countries to import the disease in the coming days. The model includes travel at different levels, which, as pointed out in Section 3.3. was a documented feature of spread.

In [170], an SIR-type metapopulation model in the GLEAM framework [30] is used that combines population densities, commute patterns and long-range travel. Used at the early stage of the spread, the authors find that it is likely that the value of the basic reproduction number $\mathcal{R}_{0}$ and the prevalence are badly estimated in some locations, with estimates in the literature at the time driven by locations with a large population. They conclude that the number of cases was probably underestimated.

\subsection{Modelling the slowing down of global spread}

In [55], a metapopulation model for the global spread of COVID-19 is used to consider in particular the role of international travel bans. The authors show that while the cordon sanitaire in Wuhan did little to slow spread within China, its impact internationally was more pronounced. The combined effect of travel restrictions and community effort is also studied, with the interesting finding that travel restrictions alone do not suffice to have an effect on propagation. In [4], a stochastic SEIR metapopulation model is used, together with Official Airlines Guide (OAG) data, to consider the role of travel restrictions taking place after 24 January 2020 . The authors found good adequation with the number of imported cases in several countries as of the end of January. They focused in particular on Australia and establish that the travel ban there might have delayed the onset of widespread propagation by four weeks. 
A stochastic simulation model is used in [69] to consider different scenarios regarding testing (rather than screening) of incoming individuals and the duration of quarantine periods. Similarly, [59] use a stochastic model to quantify the effectiveness of screening and so-called sensitisation of travellers, i.e., the provision of health information in an effort to trigger compliance with self-isolation recommendations. In [159], the example of air transportation in Brazil is considered using an SIRtype metapopulation. The speed of spread in relation to network measures such as centrality was explored, with closeness centrality shown to be a good predictor of the vulnerability of a city.

In [17], we considered the risk of disease importation in a location that is seeing little to no local transmission chains. As with most of our work on the subject, we used a modified version of the model in [23]. In this case, we used a stochastic version, which we subjected to stimulations to represent the inflow of infected individuals into a location. The model also allowed us to quantify precisely the effect of quarantine in terms of its effect on the inflow rate.

\subsection{Modelling the transition from global to local spread}

In [94], the risk of importation of COVID-19 in African countries was considered using air travel data as well as data from the Monitoring Evaluation Framework (MEF) of the WHO International Health Regulations. The model is quite simple and comprises no dynamic components, meaning that it provides a snapshot evaluation of the risk of importation. As it was formulated at the beginning of the spread event, when most of the exportation was assumed to come from China, it does nonetheless provide meaningful results.

In the already cited [17], we focused on the risk of importation of COVID19 in locations that are seeing little to no local transmission, thereby considering the interface between the rest of the world and such locations. We showed that the probability of importation was most dependent on the rate at which cases are imported in the locations, but that the outcome of a successful importation was then determined to a large extent by the intensity of public health measures in the locations.

\subsection{Models of local spread}

The location for which data became readily available the soonest was China. Since China is also a very large country, some very interesting work was carried out in the context of spread within that country. The authors of [183] considered the spread of COVID-19 within China using an interesting idea: they estimated the size of the outbreak in Wuhan from known international exportations, then used a metapopulation model with Wuhan as the source of infection to estimate spread within China. In 
[131], the role of undocumented infections is investigated in relation with the spread of COVID-19 between 372 Chinese cities using a metapopulation SEIR model incorporating documented and undocumented infections. In [112], spread within Hubei Province and in the rest of China is investigated using statistical tools (the Moran index and a logistic model). Then an ODE SEIR model is used to compute $\mathcal{R}_{0}$ in the different locations. In [174], an SEIR-type model with additional compartments for diagnosed and confirmed, suspected and infected as well as suspected but uninfected individuals is set in a metapopulation framework with two patches: Hubei Province and the rest of China. The model is used to consider the effect of lifting lockdown measures.

In [10], a simulation platform is used to consider the spread in France. The model operates at the level of subregions (départements) and supposes that individuals can be susceptible, asymptomatic, symptomatic, recovered, hospitalised and diseased. An interesting feature of the paper is a comparison between the results of continuous time deterministic and discrete stochastic methods, with the latter showing better adequation with observed data.

The authors of [46] considered spread of SARS-CoV-2 within Brazil. This colossal endeavour considers actual genotyping of the virus and prior to modelling work proper, details importations and the spatio-temporal spread of various genomes of the virus. Spatio-temporal modelling then uses a continuous phylogeographic model. The model is not predictive but sheds light on the spread process: they find that spread was mostly local, i.e., within state borders. Both within-state and between-state spread was also found to have decreased after the implementation of NPI.

In [9], the effect of heterogeneity of policies in the USA is investigated. A model is formulated that is a metapopulation in essence; based on data on people movement to places of gathering such as churches, the model allows the redistribution of individuals between locations following different types of policies. They observe that spatial heterogeneity in measures tends to increase the likelihood of subsequent infection waves. Spatial heterogeneity is also investigated in [65], which uses a metapopulation model to probe the impact of disparity of healthcare capacity in Ohio. In [53], the effect of changing travel rates within and between locations is investigated, with data for Taiwan.

Finally, note that because COVID-19 is spreading globally and that national level jurisdictions (and sometimes even lower level ones) implemented a variety of responses, it is useful to compare the situation in different jurisdictions. Even though this is not spatial modelling stricto sensu, such works are worth mentioning here as they provide the underpinning to spatial models. In [104], the authors use an SEIR model to compare transmission patterns in China, South Korea, Italy and Iran. In [103], an age-structured SEIR model is used to compare the dynamics of disease spread in Hubei Province and six European regions. The focus is on the estimation of the case-fatality (CFR), symptomatic case-fatality ( $\mathrm{SCFR}$ ) and infection-fatality (IFR) ratios. The authors find that the latter two indicators are better suited to describe the potential impact of the pandemic and note that they find geographic heterogeneity of the estimated values. This heterogeneity is not only between Hubei 
Province and the European locations under consideration, but also between the European locations themselves. With collaborators, we used the model in [23] to provide a daily forecast of spread in several Canadian provinces [21] and found that estimates for some parameters were consistent across provinces while estimates for others varied widely, in particular, the proportion of asymptomatic cases.

\subsection{Models for spread at the hyperlocal level}

A lot of work during this pandemic has focused implicitly on the hyperlocal level, but recall that here the object is models in which I found an explicit reference to spatial aspects.

In [115], a network model is used to model the spread of SARS-CoV-2 onboard the Diamond Princess cruise ship, with nodes representing individual passengers and crew members. Age-structure was used as well. The model was calibrated to known transmission data and the effect of control measures was then considered. See also the already cited [75, 143, 153, 185] for more modelling work related to spread aboard the Diamond Princess.

The authors of [43] used an SLIAR agent-based model to consider the effect of social distancing, viral shedding and what they call the social distance threshold. They find that the three lead to threshold behaviour ("phase transitions") that have different effects on the course of the epidemic.

In [81], ABM are used to consider in particular the effect of testing policies. Agents are distributed on a map depending on the population density in the areas under consideration. They are also assigned movement patterns that can cover the whole map, a medium range or a small one. Some interesting observations are that when tests have low reliability or that the ability to trace contact is low, a large fraction of the testing capacity remains unused despite an increasing incidence. They also find that mixed testing policies are useful to contain spread.

\section{New variants}

SARS-CoV-2 is an RNA virus and as such is subject to high mutation rates leading potentially to variants [72, 117]. Thus the emergence of new variants was expected from the onset of the crisis. At this point, there are several major variants to the original variant that have been detected. This number can be expected to rise: detection of most variants requires genome sequencing, which is performed at different rates in different countries [85], meaning that capacity to detect variants varies greatly globally. Of particular interest at the time of writing is B.1.1.7, which was first detected in the United Kingdom in early December 2020 but is presumed to have been spreading since as early as September 2020 . This variant is particularly concerning as it appears to be more transmissible than the original variant. It seems that this 
variant should not, however, be detrimental to the ongoing vaccination efforts [62]. Many countries took preventive measures in order to delay the arrival of the variant, essentially forbidding all travel originating from the United Kingdom, but given that circulation probably started several months before these measures, their efficacy is debatable. For instance, [71] estimates that, of 19 countries evaluated, 16 had at least a 50\% chance of having already imported the variant by 7 December 2020. The novel variants led some countries to consider exit control measures; some European Union (EU) countries (Belgium and France, for instance) decided late January 2021 or early February 2021 to forbid both entry from and exit to non-EU countries for non-essential travel. In the context of pandemic H1N1 influenza, exit screening was shown to have the potential to be more an efficacious control measure than entry screening [120]. It is therefore interesting to see this type of control finally being applied, although the intent is not the one we were advocating in [120].

Modelling the spatio-temporal spread of these novel variants can be conducted in very much the same manner as was done for the original variant. For instance, metapopulation models for multiple species such as those considered in [19, 20] can be readily adapted to a multiple variant situation. However, it is important to bear in mind that because of the detection issues mentioned earlier, these models are hard to parametrise when considering the initial spread of the variants.

\section{Discussion}

This is but a brief and very incomplete snapshot of the state of knowledge about the spread of COVID-19 at the time of writing in December 2020, with a few additional details about the new variants added in January 2021. As indicated, it is likely that I omitted a lot of publications on the subject, given the immense amount of literature COVID-19 has generated.

From the perspective of the spatio-temporal spread of the disease, although there is still much to learn, I think we also now have the luxury of hindsight: many groups, mine included, have produced a variety of models in the first few months of the crisis, which can and should now be confronted to the reality of the outbreak. Because COVID-19 is so widespread, there is less urgency to consider its spatial spread in the perspective of emergency response and the focus could now evolve, at least in part, to the evaluation of the models we produced. The problem of reimportation of the disease in locations having managed to drive it away remains an important one, so I am not advocating to stop all work regarding spatial spread; I am only pointing out that understanding what worked and what did not during the initial spread would actually help for these subsequent importation events.

Going forward, though, I believe that there is still a lot to be done on one key aspect of spatio-temporal models: most of the work carried out by those of us working in this area has come to rely on one particular dataset, the so-called IATA air transport data. Figure 2 shows that in the particular instance of COVID-19, the quality of this data leaves a lot to be desired. When the data for 2020 becomes available in 
2021, it will be extremely important to scrutinise it in order to understand what type of changes took place. Another important point to ponder will be the use of other data sources to compensate for this loss of relevance of IATA-type data. Cell phone location data is showing promise, but it suffers from several limitations, the most important of which being that it is most useful and detailed at the country level and, more importantly, that it is either proprietary or extremely expensive to acquire.

Acknowledgements I am supported in part by NSERC and by CIHR through the Canadian COVID19 Mathematical Modelling Task Force. I acknowledge support both financial and logistical from the Public Health Agency of Canada.

\section{References}

1. 20 minutes avec AFP. Coronavirus: Trois cas confirmés en France, 15 nouveaux décès en Chine. 20 minutes, January 2020. https://www.20minutes.fr/sante/ 2703111-20200125-coronavirus-trois-cas-confirmes-france-15-nouveaux-deces-chine

2. Z. Abdullah and H. Salamat. https://www.channelnewsasia.com/news/singapore/wuhanvirus-pneumonia-singapore-confirms-first-case-12312860. CNA Asia, January 2020. https://www.channelnewsasia.com/news/singapore/ wuhan-virus-pneumonia-singapore-confirms-first-case-12312860

3. P. Abry, N. Pustelnik, S. Roux, et al. Spatial and temporal regularization to estimate COVID19 reproduction number $\mathrm{R}(\mathrm{t})$ : Promoting piecewise smoothness via convex optimization. PloS One, 15:e0237901, 2020.

4. A. Adekunle, M. Meehan, D. Rojas-Alvarez, et al. Delaying the COVID-19 epidemic in Australia: evaluating the effectiveness of international travel bans. Australian and New Zealand Journal of Public Health, July 2020.

5. A. Adiga, S. Chu, S. Eubank, et al. Disparities in spread and control of influenza in slums of Delhi: findings from an agent-based modelling study. BMJ Open, 8:e017353, January 2018.

6. A. Adiga, S. Venkatramanan, J. Schlitt, et al. Evaluating the impact of international airline suspensions on the early global spread of COVID-19. medRxiv, February 2020.

7. M. Ajelli, B. Gonçalves, D. Balcan, et al. Comparing large-scale computational approaches to epidemic modeling: agent-based versus structured metapopulation models. BMC Infectious Diseases, 10:190, June 2010.

8. M. E. Alexander and R. Kobes. Effects of vaccination and population structure on influenza epidemic spread in the presence of two circulating strains. BMC Public Health, 11 Suppl $1:$ S8, February 2011.

9. B. M. Althouse, B. Wallace, B. Case, et al. The unintended consequences of inconsistent pandemic control policies. medRxiv, August 2020.

10. P. Amar. Pandæsim: an epidemic spreading stochastic simulator. Biology, 9, September 2020.

11. J. J. Angulo, C. K. Takiguti, C. A. Pederneiras, et al. Identification of pattern and process in the spread of a contagious disease. Social Science \& Medicine, 13D:183-189, November 1979.

12. A. Apolloni, C. Poletto, and V. Colizza. Age-specific contacts and travel patterns in the spatial spread of 2009 H1N1 influenza pandemic. BMC Infectious Diseases, 13:176, April 2013.

13. J. Arino. Mathematical epidemiology in a data-rich world. Infectious Disease Modelling, $5: 161-188,2020$

14. J. Arino, A. Ducrot, and P. Zongo. A metapopulation model for malaria with transmissionblocking partial immunity in hosts. Journal of Mathematical Biology, 64(3):423-448, 2012.

15. J. Arino and P. van den Driessche. A multi-city epidemic model. Mathematical Population Studies, 10(3):175-193, 2003. 
16. J. Arino. Spatio-temporal spread of infectious pathogens of humans. Infectious Disease Modelling, 2:218-228, May 2017.

17. J. Arino, N. Bajeux, S. Portet, and J. Watmough. Assessing the risk of COVID-19 importation and the effect of quarantine. medRxiv, 2020.

18. J. Arino, F. Brauer, P. van den Driessche, et al. Simple models for containment of a pandemic. Journal of the Royal Society, Interface, 3:453-457, June 2006.

19. J. Arino, J. R. Davis, D. Hartley, et al. A multi-species epidemic model with spatial dynamics. Mathematical Medicine and Biology, 22:129-142, June 2005.

20. J. Arino, R. Jordan, and P. van den Driessche. Quarantine in a multi-species epidemic model with spatial dynamics. Mathematical Biosciences, 206:46-60, March 2007.

21. J. Arino, S. Portet, N. Bajeux, and A. Ciupeanu. Investigation of global and local COVID-19 importation risks. Technical report, Public Health Agency of Canada, 2020.

22. J. Arino and S. Portet. Epidemiological implications of mobility between a large urban centre and smaller satellite cities. Journal of Mathematical Biology, 71(5):1243-1265, 2015.

23. J. Arino and S. Portet. A simple model for COVID-19. Infectious Disease Modelling, 5:309-315, 2020

24. J. Arino, S. Portet, and E. Rees. A method for assessing the most likely next destinations of COVID-19. In preparation.

25. J. Arino, C. Sun, and W. Yang. Revisiting a two-patch SIS model with infection during transport. Mathematical Medicine and Biology, 33(1):29-55, 2016.

26. E. Asano, L. J. Gross, S. Lenhart, and L. A. Real. Optimal control of vaccine distribution in a rabies metapopulation model. Mathematical Biosciences and Engineering, 5:219-238, April 2008.

27. Australian Government Department of Health. First confirmed case of novel coronavirus in Australia. Media release, January 2020. https: //www.health.gov. au/ministers/the-hon-greg-hunt-mp/media/ first-confirmed-case-of-novel-coronavirus-in-australia

28. E. I. Azhar, S. Lanini, G. Ippolito, and A. Zumla. The Middle East Respiratory Syndrome coronavirus - a continuing risk to global health security. Advances in Experimental Medicine and Biology, 972:49-60, 2017.

29. A. S. Azman and J. Lessler. Reactive vaccination in the presence of disease hotspots. Proceedings. Biological Sciences, 282:20141341, January 2015.

30. D. Balcan, B. Gonçalves, H. Hu, et al. Modeling the spatial spread of infectious diseases: the GLobal Epidemic and Mobility computational model. Journal of Computational Science, 1(3):132-145, 2010.

31. F. Ball, T. Britton, T. House, et al. Seven challenges for metapopulation models of epidemics, including households models. Epidemics, 10:63-67, March 2015.

32. F. Ball and T. House. Heterogeneous network epidemics: real-time growth, variance and extinction of infection. Journal of Mathematical Biology, 75:577-619, September 2017.

33. D. H. Barmak, C. O. Dorso, M. Otero, and H. G. Solari. Dengue epidemics and human mobility. Physical Review E, 84:011901, July 2011.

34. R. C. Barnard, L. Berthouze, P. L. Simon, and I. Z. Kiss. Epidemic threshold in pairwise models for clustered networks: closures and fast correlations. Journal of Mathematical Biology, 79:823-860, August 2019.

35. A. Bastola, R. Sah, A. J. Rodriguez-Morales, et al. The first 2019 novel coronavirus case in Nepal. The Lancet Infectious diseases, 20:279-280, March 2020.

36. M. M. Böhmer, U. Buchholz, V. M. Corman, et al. Investigation of a COVID-19 outbreak in Germany resulting from a single travel-associated primary case: a case series. The Lancet Infectious Diseases, 20:920-928, August 2020.

37. D. Bichara and A. Iggidr. Multi-patch and multi-group epidemic models: a new framework. Journal of Mathematical Biology, 77:107-134, July 2018.

38. N. Bifolchi, R. Deardon, and Z. Feng. Spatial approximations of network-based individual level infectious disease models. Spatial and Spatio-Temporal Epidemiology, 6:59-70, September 2013. 
39. L. I. Birtolo, V. Maestrini, P. Severino, et al. Coronavirus disease 2019 in Rome: was it circulating before December? Journal of Cardiovascular Medicine, 21:835-836, October 2020.

40. D. Bitar, A. Goubar, and J. C. Desenclos. International travels and fever screening during epidemics: a literature review on the effectiveness and potential use of non-contact infrared thermometers. Euro Surveillance, 14(2):1-5, February 2009.

41. W. Bock and Y. Jayathunga. Optimal control of a multi-patch Dengue model under the influence of Wolbachia bacterium. Mathematical Biosciences, 315:108219, September 2019.

42. J. T. Bowen and C. Laroe. Airline networks and the international diffusion of severe acute respiratory syndrome (SARS). The Geographical Journal, 172:130-144, June 2006.

43. B. Braun, B. Taraktaş, B. Beckage, and J. Molofsky. Simulating phase transitions and control measures for network epidemics caused by infections with presymptomatic, asymptomatic, and symptomatic stages. PLoS One, 15:e0238412, 2020.

44. D. Brockmann and D. Helbing. The hidden geometry of complex, network-driven contagion phenomena. Science, 342:1337-1342, December 2013.

45. G. M. Bwire and L. S. Paulo. Coronavirus disease-2019: is fever an adequate screening for the returning travelers? Tropical Medicine and Health, 48:14, 2020.

46. D. S. Candido, I. M. Claro, J. G. de Jesus, et al. Evolution and epidemic spread of SARSCoV-2 in Brazil. Science, 369:1255-1260, September 2020.

47. Z. Cao, D. Zeng, X. Zheng, et al. Spatio-temporal evolution of Beijing 2003 SARS epidemic. Science China. Earth Sciences, 53:1017-1028, 2010.

48. N. B. Carnegie. Effects of contact network structure on epidemic transmission trees: implications for data required to estimate network structure. Statistics in Medicine, 37:236-248, January 2018.

49. C. Carpenter and L. Sattenspiel. The design and use of an agent-based model to simulate the 1918 influenza epidemic at Norway House, Manitoba. American Journal of Human Biology, 21:290-300, 2009.

50. S. Chadsuthi, B. M. Althouse, S. Iamsirithaworn, et al. Travel distance and human movement predict paths of emergence and spatial spread of chikungunya in Thailand. Epidemiology \& Infection, 146:1654-1662, October 2018.

51. D. Champredon, M. Najafi, M. Laskowski, et al. Individual movements and contact patterns in a Canadian long-term care facility. AIMS Public Health, 5:111-121, 2018.

52. M. Chan-Yeung and R.-H. Xu. SARS: epidemiology. Respirology, 8 Suppl:S9-14, November 2003.

53. M.-C. Chang, R. Kahn, Y.-A. Li, et al. Variation in human mobility and its impact on the risk of future COVID-19 outbreaks in Taiwan. medRxiv, August 2020.

54. J. D. Cherry. The chronology of the 2002-2003 SARS mini pandemic. Paediatric Respiratory Reviews, 5:262-269, December 2004

55. M. Chinazzi, J. T. Davis, M. Ajelli, et al. The effect of travel restrictions on the spread of the 2019 novel coronavirus (covid-19) outbreak. Science, 368:395-400, April 2020.

56. G. Chowell, J. M. Hyman, S. Eubank, and C. Castillo-Chavez. Scaling laws for the movement of people between locations in a large city. Physical Review E, 68:066102, December 2003.

57. G. Chowell, L. Sattenspiel, S. Bansal, and C. Viboud. Mathematical models to characterize early epidemic growth: A review. Physics of Life Reviews, 18:66-97, September 2016.

58. P. Christidis and A. Christodoulou. The predictive capacity of air travel patterns during the global spread of the COVID-19 pandemic: risk, uncertainty and randomness. International Journal of Environmental Research and Public Health, 17, May 2020.

59. S. Clifford, C. A. B. Pearson, P. Klepac, et al. Effectiveness of interventions targeting air travellers for delaying local outbreaks of SARS-CoV-2. Journal of Travel Medicine, 27, August 2020.

60. J. Coleman. Vietnam reports first coronavirus cases. The Hill, January 2020.

61. V. Colizza, M. Barthélemy, A. Barrat, and A. Vespignani. Epidemic modeling in complex realities. Comptes rendus Biologies, 330:364-374, April 2007. 
62. P. Conti, A. Caraffa, C. E. Gallenga, et al. The British variant of the new coronavirus-19 (Sars-Cov-2) should not create a vaccine problem. Journal of Biological Regulators and Homeostatic Agents, 35, December 2020.

63. S. Copiello and C. Grillenzoni. The spread of 2019-nCoV in China was primarily driven by population density. Comment on "Association between short-term exposure to air pollution and COVID-19 infection: Evidence from China" by Zhu et al. The Science of the Total Environment, 744:141028, November 2020.

64. G. Cruz-Pacheco, J. F. Bustamante-Castañeda, J. G. Caputo, et al. Dispersion of a new coronavirus SARS-CoV-2 by airlines in 2020: temporal estimates of the outbreak in mexico. Revista de Investigacion Clinica, 72:138-143, 2020.

65. D. F. Cuadros, Y. Xiao, Z. Mukandavire, et al. Spatiotemporal transmission dynamics of the covid-19 pandemic and its impact on critical healthcare capacity. Health \& Place, 64:102404, July 2020.

66. O. O. Dalgıç, O. Y. Özaltın, W. A. Ciccotelli, and F. S. Erenay. Deriving effective vaccine allocation strategies for pandemic influenza: Comparison of an agent-based simulation and a compartmental model. PLoS One, 12:e0172261, 2017.

67. M. Dell'Omodarme and M. C. Prati. The probability of failing in detecting an infectious disease at entry points into a country. Statistics in Medicine, 24:2669-2679, September 2005.

68. A. Deslandes, V. Berti, Y. Tandjaoui-Lambotte, et al. SARS-CoV-2 was already spreading in France in late December 2019. International Journal of Antimicrobial Agents, 55:106006, June 2020.

69. B. L. Dickens, J. R. Koo, J. T. Lim, et al. Strategies at points of entry to reduce importation risk of COVID-19 cases and re-open travel. Journal of Travel Medicine, August 2020.

70. M. M. Dickson, G. Espa, D. Giuliani, et al. Assessing the effect of containment measures on the spatio-temporal dynamic of COVID-19 in Italy. Nonlinear Dynamics, pages 1-14, August 2020.

71. Z. Du, L. Wang, B. Yang, et al. International risk of the new variant COVID-19 importations originating in the United Kingdom. medRxiv : the preprint server for health sciences, January 2021.

72. E. A. Duarte, I. S. Novella, S. C. Weaver, et al. RNA virus quasispecies: significance for viral disease and epidemiology. Infectious Agents and Disease, 3:201-214, August 1994.

73. M. C. Eisenberg, Z. Shuai, J. H. Tien, and P. van den Driessche. A cholera model in a patchy environment with water and human movement. Mathematical Biosciences, 246:105-112, November 2013.

74. A. Elengoe. COVID-19 outbreak in Malaysia. Osong Public Health and Research Perspectives, 11:93-100, June 2020.

75. J. C. Emery, T. W. Russell, Y. Liu, et al. The contribution of asymptomatic SARS-CoV-2 infections to transmission on the Diamond Princess cruise ship. eLife, 9, August 2020.

76. J. M. Epstein. Generative Social Science: Studies in Agent-Based Computational Modeling, volume 13. Princeton University Press, 2006.

77. S. Eubank. Network based models of infectious disease spread. Japanese Journal of Infectious Diseases, 58:S9-13, December 2005.

78. C. Fan, L. Liu, W. Guo, et al. Prediction of epidemic spread of the 2019 novel coronavirus driven by Spring Festival transportation in China: a population-based study. International Journal of Environmental Research and Public Health, 17, March 2020.

79. H. Fang, J. Chen, and J. Hu. Modelling the SARS epidemic by a lattice-based Monte-Carlo simulation. Annual International Conference of the IEEE Engineering in Medicine and Biology Society, 2005:7470-7473, 2005.

80. J. R. Fauver, M. E. Petrone, E. B. Hodcroft, et al. Coast-to-coast spread of SARS-CoV-2 during the early epidemic in the United States. Cell, 181:990-996.e5, May 2020.

81. V. G. Fiore, N. DeFelice, B. S. Glicksberg, et al. Containment of future waves of COVID-19: simulating the impact of different policies and testing capacities for contact tracing, testing, and isolation. medRxiv, June 2020. 
82. S. M. Firestone, M. P. Ward, R. M. Christley, and N. K. Dhand. The importance of location in contact networks: Describing early epidemic spread using spatial social network analysis. Preventive Veterinary Medicine, 102:185-195, December 2011.

83. A. Flahault, S. Letrait, P. Blin, et al. Modelling the 1985 influenza epidemic in France. Statistics in Medicine, 7:1147-1155, November 1988.

84. C. M. C. B. Fortaleza, R. B. Guimarães, G. B. de Almeida, et al. Taking the inner route: spatial and demographic factors affecting vulnerability to COVID-19 among 604 cities from inner São Paulo State, Brazil. Epidemiology \& Infection, 148:e118, June 2020.

85. Y. Furuse. Genomic sequencing effort for SARS-CoV-2 by country during the pandemic. International Journal of Infectious Diseases, 103:305 - 307, 2021.

86. W. Gaber, U. Goetsch, R. Diel, et al. Screening for infectious diseases at international airports: the Frankfurt model. Aviation, Space, and Environmental Medicine, 80:595-600, July 2009.

87. D. Gao and S. Ruan. An SIS patch model with variable transmission coefficients. Mathematical Biosciences, 232:110-115, August 2011.

88. D. Gao and S. Ruan. A multi-patch malaria model with logistic growth populations. SIAM Journal on Applied Mathematics, 72:819-841, January 2012.

89. D. Gao, P. van den Driessche, and C. Cosner. Habitat fragmentation promotes malaria persistence. Journal of Mathematical Biology, 79:2255-2280, December 2019.

90. L. M. Gardner, A. A. Chughtai, and C. R. MacIntyre. Risk of global spread of Middle East respiratory syndrome coronavirus (MERS-CoV) via the air transport network. Journal of Travel Medicine, 23, June 2016.

91. E. Gayawan, O. O. Awe, B. M. Oseni, et al. The spatio-temporal epidemic dynamics of COVID-19 outbreak in Africa. Epidemiology \& Infection, 148:e212, September 2020.

92. K. Gaythorpe and B. Adams. Disease and disaster: Optimal deployment of epidemic control facilities in a spatially heterogeneous population with changing behaviour. Journal of Theoretical Biology, 397:169-178, May 2016.

93. J. L. Geoghegan, A. F. Saavedra, S. Duchêne, et al. Continental synchronicity of human influenza virus epidemics despite climatic variation. PLoS Pathogens, 14:e1006780, January 2018.

94. M. Gilbert, G. Pullano, F. Pinotti, et al. Preparedness and vulnerability of African countries against importations of COVID-19: a modelling study. The Lancet, 395:871-877, March 2020.

95. K. Glass and B. Barnes. Eliminating infectious diseases of livestock: a metapopulation model of infection control. Theoretical Population Biology, 85:63-72, May 2013.

96. Q. Griette, G. Raoul, and S. Gandon. Virulence evolution at the front line of spreading epidemics. Evolution, 69:2810-2819, November 2015.

97. S. S. Gunthe and S. S. Patra. Impact of international travel dynamics on domestic spread of 2019-nCoV in India: origin-based risk assessment in importation of infected travelers. Globalization and Health, 16:45, May 2020.

98. D. Guo, K. C. Li, T. R. Peters, et al. Multi-scale modeling for the transmission of influenza and the evaluation of interventions toward it. Scientific Reports, 5:8980, March 2015.

99. C. M. Hafner. The spread of the Covid-19 pandemic in time and space. International Journal of Environmental Research and Public Health, 17, May 2020.

100. N. Haider, A. Yavlinsky, Y.-M. Chang, et al. The Global Health Security Index and Joint External Evaluation score for health preparedness are not correlated with countries' COVID19 detection response time and mortality outcome. Epidemiology \& Infection, 148:e210, September 2020.

101. I. M. Hall, J. R. Egan, I. Barrass, et al. Comparison of smallpox outbreak control strategies using a spatial metapopulation model. Epidemiology \& Infection, 135:1133-1144, October 2007.

102. P. Harvim, H. Zhang, P. Georgescu, and L. Zhang. Transmission dynamics and control mechanisms of vector-borne diseases with active and passive movements between urban and satellite cities. Bulletin of Mathematical Biology, 81:4518-4563, November 2019. 
103. A. Hauser, M. J. Counotte, C. C. Margossian, et al. Estimation of SARS-CoV-2 mortality during the early stages of an epidemic: A modeling study in Hubei, China, and six regions in Europe. PLoS Medicine, 17:e1003189, July 2020.

104. J. He, G. Chen, Y. Jiang, et al. Comparative infection modeling and control of COVID19 transmission patterns in China, South Korea, Italy and Iran. The Science of the Total Environment, 747:141447, August 2020.

105. A. Hernandez-Matamoros, H. Fujita, T. Hayashi, and H. Perez-Meana. Forecasting of COVID19 per regions using ARIMA models and polynomial functions. Applied Soft Computing, 96:106610, November 2020.

106. A. Hohl, E. M. Delmelle, M. R. Desjardins, and Y. Lan. Daily surveillance of COVID-19 using the prospective space-time scan statistic in the United States. Spatial and Spatio-Temporal Epidemiology, 34:100354, August 2020.

107. M. L. Holshue, C. DeBolt, S. Lindquist, et al. First case of 2019 novel coronavirus in the United States. The New England Journal of Medicine, 382:929-936, March 2020.

108. D. Holtz, M. Zhao, S. G. Benzell, et al. Interdependence and the cost of uncoordinated responses to covid-19. Proceedings of the National Academy of Sciences of the United States of America, 117:19837-19843, August 2020.

109. T. Hornbeck, D. Naylor, A. M. Segre, et al. Using sensor networks to study the effect of peripatetic healthcare workers on the spread of hospital-associated infections. The Journal of Infectious Diseases, 206:1549-1557, November 2012.

110. B. Hu, J. Gong, J. Sun, and J. Zhou. Exploring the epidemic transmission network of SARS in-out flow in mainland China. Chinese Science Bulletin, 58:1818-1831, 2013.

111. B. Hu, J. Gong, J. Zhou, et al. Spatial-temporal characteristics of epidemic spread in-out flow-using SARS epidemic in Beijing as a case study. Science China. Earth Sciences, 56:1380-1397, 2013.

112. R. Huang, M. Liu, and Y. Ding. Spatial-temporal distribution of COVID-19 in China and its prediction: A data-driven modeling analysis. Journal of Infection in Developing Countries, 14:246-253, March 2020.

113. O. Iritani, T. Okuno, D. Hama, et al. Clusters of COVID-19 in long-term care hospitals and facilities in Japan from 16 January to 9 May 2020. Geriatrics \& Gerontology International, 20:715-719, July 2020.

114. ISO. Iso 3166 code lists, 2013.

115. S. M. Jenness, K. S. Willebrand, A. A. Malik, et al. Modeling dynamic network strategies for SARS-CoV-2 control on a cruise ship. medRxiv, September 2020.

116. E. Karimi, K. Schmitt, and A. Akgunduz. Effect of individual protective behaviors on influenza transmission: an agent-based model. Health Care Management Science, 18:318333, September 2015.

117. T. F. Kautz and N. L. Forrester. RNA virus fidelity mutants: a useful tool for evolutionary biology or a complex challenge? Viruses, 10, November 2018.

118. M. R. Kelly, J. H. Tien, M. C. Eisenberg, and S. Lenhart. The impact of spatial arrangements on epidemic disease dynamics and intervention strategies. Journal of Biological Dynamics, 10:222-249, 2016.

119. K. Khan, J. Arino, W. Hu, et al. Spread of a novel influenza A (H1N1) virus via global airline transportation. The New England Journal of Medicine, 361:212-214, July 2009.

120. K. Khan, R. Eckhardt, J. S. Brownstein, et al. Entry and exit screening of airline travellers during the A(H1N1) 2009 pandemic: a retrospective evaluation. Bulletin of the World Health Organization, 91:368-376, May 2013.

121. A. Khatua, T. K. Kar, S. K. Nandi, et al. Impact of human mobility on the transmission dynamics of infectious diseases. Energy, Ecology \& Environment, pages 1-18, May 2020.

122. J. E. Kim, H. Lee, C. H. Lee, and S. Lee. Assessment of optimal strategies in a two-patch dengue transmission model with seasonality. PloS One, 12:e0173673, 2017.

123. P. Klepac, I. Megiddo, B. T. Grenfell, and R. Laxminarayan. Self-enforcing regional vaccination agreements. Journal of the Royal Society, Interface, 13:20150907, January 2016.

124. M. U. G. Kraemer, C.-H. Yang, B. Gutierrez, et al. The effect of human mobility and control measures on the COVID-19 epidemic in China. Science, 368:493-497, May 2020. 
125. C. Kuhn and A. Rose. Tall Pines Healthcare COVID-19 outbreak experience in rural Waldo County, Maine, April 2020. The Journal of Ambulatory Care Management, 43:294-300, 2020.

126. S. Kumar, J. J. Grefenstette, D. Galloway, et al. Policies to reduce influenza in the workplace: impact assessments using an agent-based model. American Journal of Public Health, 103:1406-1411, August 2013.

127. S. Kumar, K. Piper, D. D. Galloway, et al. Is population structure sufficient to generate area-level inequalities in influenza rates? An examination using agent-based models. BMC Public Health, 15:947, September 2015.

128. G. La Rosa, P. Mancini, G. Bonanno Ferraro, et al. SARS-CoV-2 has been circulating in northern Italy since December 2019: Evidence from environmental monitoring. The Science of the Total Environment, 750:141711, August 2020.

129. M. Laskowski, V. R. Duvvuri, D. L. Buckeridge, et al. Influenza H3N2 variant viruses with pandemic potential: preventing catastrophe in remote and isolated Canadian communities. Preventive Medicine, 57:910-913, December 2013.

130. S. Lee and C. Castillo-Chavez. The role of residence times in two-patch dengue transmission dynamics and optimal strategies. Journal of Theoretical Biology, 374:152-164, June 2015.

131. R. Li, S. Pei, B. Chen, et al. Substantial undocumented infection facilitates the rapid dissemination of novel coronavirus (SARS-CoV-2). Science, 368:489-493, May 2020.

132. X. Liu, X. Chen, and Y. Takeuchi. Dynamics of an SIQS epidemic model with transportrelated infection and exit-entry screenings. Journal of Theoretical Biology, 285:25-35, September 2011.

133. X. Liu and Y. Takeuchi. Spread of disease with transport-related infection and entry screening. Journal of Theoretical Biology, 242:517-528, September 2006.

134. I. M. Longini, A. Nizam, S. Xu, et al. Containing pandemic influenza at the source. Science, 309:1083-1087, August 2005.

135. L. Matrajt, M. E. Halloran, and I. M. Longini. Optimal vaccine allocation for the early mitigation of pandemic influenza. PLoS Computational Biology, 9:e1002964, 2013.

136. L. Matthews, D. T. Haydon, D. J. Shaw, et al. Neighbourhood control policies and the spread of infectious diseases. Proceedings. Biological Sciences, 270:1659-1666, August 2003.

137. T. M. McMichael, S. Clark, S. Pogosjans, et al. COVID-19 in a Long-Term Care Facility - King County, Washington, February 27-March 9, 2020. Morbidity and Mortality Weekly Report, 69:339-342, March 2020.

138. P. Melin, J. C. Monica, D. Sanchez, and O. Castillo. Analysis of spatial spread relationships of coronavirus (COVID-19) pandemic in the world using self organizing maps. Chaos, Solitons, and Fractals, 138:109917, September 2020.

139. L. A. Meyers, M. E. J. Newman, and B. Pourbohloul. Predicting epidemics on directed contact networks. Journal of Theoretical Biology, 240:400-418, June 2006.

140. L. A. Meyers, B. Pourbohloul, M. E. J. Newman, et al. Network theory and SARS: predicting outbreak diversity. Journal of Theoretical Biology, 232:71-81, January 2005.

141. A. Mishra, B. Ambrosio, S. Gakkhar, and M. A. Aziz-Alaoui. A network model for control of dengue epidemic using sterile insect technique. Mathematical Biosciences and Engineering, 15:441-460, April 2018.

142. S. Mishra, A. Mohapatra, R. Kumar, et al. Restricting rural-urban connect to combat infectious disease epidemic as India fights COVID-19. Journal of Family Medicine and Primary Care, 9:1792-1794, April 2020.

143. K. Mizumoto and G. Chowell. Transmission potential of the novel coronavirus (COVID-19) onboard the Diamond Princess Cruises ship. Infectious Disease Modelling, 5:264-270, 2020.

144. A. Mollalo, K. M. Rivera, and B. Vahedi. Artificial neural network modeling of novel coronavirus (COVID-19) incidence rates across the continental United States. International Journal of Environmental Research and Public Health, 17, June 2020.

145. D. Mollison. Spatial contact models for ecological and epidemic spread. Journal of the Royal Statistical Society: Series B (Methodological), 39(3):283-313, 1977.

146. D. Mollison. Markovian contact processes. Advances in Applied Probability, 10(1):85-108, 1978. 
147. D. Mollison and K. Kuulasmaa. Spatial epidemic models: theory and simulations. Population Dynamics of Rabies in Wildlife, 8:291-309, 1985.

148. R. Moss, E. Naghizade, M. Tomko, and N. Geard. What can urban mobility data reveal about the spatial distribution of infection in a single city? BMC Public Health, 19:656, May 2019.

149. V. A. Mouchtouri, E. P. Christoforidou, M. An der Heiden, et al. exit and entry screening practices for infectious diseases among travelers at points of entry: looking for evidence on public health impact. International Journal of Environmental Research and Public Health, 16, November 2019.

150. M. Najafi, M. Laskowski, P. T. de Boer, et al. The effect of individual movements and interventions on the spread of Influenza in long-term care facilities. Medical Decision Making, 37:871-881, November 2017.

151. New Straits Times. 3 coronavirus cases confirmed in Johor Baru. New Straits Times, January 2020. https://wwW.nst.com.my/news/nation/2020/01/559563/ breaking-3-coronavirus-cases-confirmed-johor-baru

152. T. H. D. Nguyen and D. C. Vu. Summary of the COVID-19 outbreak in Vietnam - Lessons and suggestions. Travel Medicine and Infectious Disease, 37:101651, 2020.

153. H. Nishiura. Backcalculating the incidence of infection with COVID-19 on the Diamond Princess. Journal of Clinical Medicine, 9, February 2020.

154. Y. Ohkusa and T. Sugawara. Application of an individual-based model with real data for transportation mode and location to pandemic influenza. Journal of Infection and Chemotherapy, 13:380-389, December 2007.

155. Y. Ohkusa and T. Sugawara. Simulation model of pandemic influenza in the whole of Japan. Japanese Journal of Infectious Diseases, 62:98-106, March 2009.

156. B. Pourbohloul, A. Ahued, B. Davoudi, et al. Initial human transmission dynamics of the pandemic (H1N1) 2009 virus in North America. Influenza and Other Respiratory Viruses, 3:215-222, September 2009.

157. G. Pullano, F. Pinotti, E. Valdano, et al. Novel coronavirus (2019-nCoV) early-stage importation risk to Europe, January 2020. Euro Surveillance, 25, January 2020.

158. L. Rass and J. Radcliffe. Spatial Deterministic Epidemics, volume 102 of Mathematical Surveys and Monographs. American Mathematical Society, 2003.

159. S. P. Ribeiro, A. Castro E Silva, W. Dáttilo, et al. Severe airport sanitarian control could slow down the spreading of covid-19 pandemics in brazil. PeerJ, 8:e9446, 2020.

160. S. Riley. Large-scale spatial-transmission models of infectious disease. Science, 316:12981301, June 2007.

161. D. A. Robertson. Spatial transmission models: A taxonomy and framework. Risk Analysis, 39:225-243, January 2019.

162. S. Ruan, W. Wang, and S. A. Levin. The effect of global travel on the spread of SARS. Mathematical Biosciences and Engineering, 3:205-218, January 2006.

163. T. W. Russell, J. Hellewell, C. I. Jarvis, et al. Estimating the infection and case fatality ratio for coronavirus disease (COVID-19) using age-adjusted data from the outbreak on the Diamond Princess cruise ship, February 2020. Euro Surveillance, 25, March 2020.

164. L. A. Rvachev and I. M. Longini Jr. A mathematical model for the global spread of influenza. Mathematical Biosciences, 75(1):3-22, 1985.

165. Q. Shao and M. Jia. Influences on influenza transmission within terminal based on hierarchical structure of personal contact network. BMC Public Health, 15:257, March 2015.

166. S. M. Shi, I. Bakaev, H. Chen, et al. Risk factors, presentation, and course of Coronavirus Disease 2019 in a large, academic long-term care facility. Journal of the American Medical Directors Association, 21:1378-1383.e1, October 2020.

167. C. D. Shrader, S. Assadzandi, C. S. Pilkerton, and A. M. Ashcraft. Responding to a COVID-19 outbreak at a long-term care facility. Journal of Applied Gerontology, page 733464820959163, September 2020.

168. W. Sim. Japan confirms first case of infection from Wuhan coronavirus; Vietnam quarantines two tourists. The Straits Times, January 2020. 
169. M. Singh, P. Sarkhel, G. J. Kang, et al. Impact of demographic disparities in social distancing and vaccination on influenza epidemics in urban and rural regions of the United States. BMC Infectious Diseases, 19:221, March 2019.

170. M. Siwiak, P. Szczesny, and M. Siwiak. From the index case to global spread: the global mobility based modelling of the COVID-19 pandemic implies higher infection rate and lower detection ratio than current estimates. PeerJ, 8:e9548, 2020.

171. J. Snow. On the Mode of Communication of Cholera. John Churchill, second edition, 1855.

172. N. M. Stall, A. Jones, K. A. Brown, et al. For-profit long-term care homes and the risk of COVID-19 outbreaks and resident deaths. Canadian Medical Association Journal, 192:E946E955, August 2020.

173. C. Sun, W. Yang, J. Arino, and K. Khan. Effect of media-induced social distancing on disease transmission in a two patch setting. Mathematical Biosciences, 230:87-95, April 2011.

174. X. Sun, Y. Xiao, and X. Ji. When to lift the lockdown in Hubei province during COVID-19 epidemic? an insight from a patch model and multiple source data. Journal of Theoretical Biology, 507:110469, August 2020.

175. C. T. Telford, U. Onwubiko, D. P. Holland, et al. Preventing COVID-19 outbreaks in longterm care facilities through preemptive testing of residents and staff members - Fulton County, Georgia, March-May 2020. Morbidity and Mortality Weekly Report, 69:1296-1299, September 2020 .

176. Thucydides. The History of the Peloponnesian War. Circa 430 BCE. http://classics.mit.edu/Thucydides/pelopwar.html

177. T. N. Vilches, M. Jaberi-Douraki, and S. M. Moghadas. Risk of influenza infection with low vaccine effectiveness: the role of avoidance behaviour. Epidemiology \& Infection, 147:e75, January 2019.

178. X. Wang, S. Liu, L. Wang, and W. Zhang. An epidemic patchy model with entry-exit screening. Bulletin of Mathematical Biology, 77:1237-1255, July 2015.

179. World Health Organization. Novel Coronavirus - Japan (ex-China). Online, January 2020.

180. World Health Organization. Novel Coronavirus - Republic of Korea (ex-China). Online, January 2020.

181. World Health Organization. Novel Coronavirus - Thailand (ex-China). Online, January 2020.

182. World Health Organization. Novel Coronavirus (2019-nCoV) - Situation report 4. Online, January 2020. 24 January 2020.

183. J. T. Wu, K. Leung, and G. M. Leung. Nowcasting and forecasting the potential domestic and international spread of the 2019-nCoV outbreak originating in Wuhan, China: a modelling study. The Lancet, 395:689-697, February 2020.

184. Z. Xie, Y. Qin, Y. Li, et al. Spatial and temporal differentiation of COVID-19 epidemic spread in mainland China and its influencing factors. The Science of the Total Environment, 744:140929, November 2020.

185. S. Zhang, M. Diao, W. Yu, et al. Estimation of the reproductive number of novel coronavirus (COVID-19) and the probable outbreak size on the Diamond Princess cruise ship: A datadriven analysis. International Journal of Infectious Diseases, 93:201-204, April 2020. 